\title{
Preface to the special issue on advances in Spatio-temporal data analysis and management
}

\author{
Kai Zheng ${ }^{1} \cdot$ Feifei $\mathrm{Li}^{2} \cdot$ Kyuseok Shim $^{3}$
}

Published online: 2 October 2017

(C) Springer Science+Business Media, LLC 2017

We are delighted to present this special issue of Geoinformatica on Advances in SpatioTemporal Data Analysis and Management. Recent years have witnessed the explosion of spatio-temporal data generated from a wide range of enterprises and applications at an unprecedented way. New methods and trends in mobility data management, geographic knowledge discovery, semantic modeling, spatial data mining, and geo-visualization - to name a few - have drastically changed the way spatio-temporal data are collected, modeled, managed, and analyzed.

The aim of this special issue is to investigate the recent development of new technologies, platforms, and frameworks that can support scalable query and analysis over a variety of spatio-temporal data types. The guest editors selected 3 contributions covers varying topics within this theme.

The first article, by Zhang et al. on " Efficient Evaluation of Shortest Travel-time Path Queries through Spatial Mashups" proposes cost-effective way for LBS providers to estimate travel time for routes by issuing external route requests to Web mapping services and takes advantage of direction sharing, parallel requesting and waypoints supported by Web mapping services to reduce the number of external route requests and the query response time for shortest travel-time route queries.

Kai Zheng

zhengkai@uestc.edu.cn

Feifei Li

lifeifei@cs.utah.edu

Kyuseok Shim

kshim@snu.ac.kr

1 University of Electronic Science and Technology of China, Chengdu, China

2 University of Utah, Salt Lake City, UT 84112, USA

3 Seoul National University, Seoul, South Korea 
In "EPLA:Efficient Personal Location Anonymity", the authors develop a an efficient method, namely EPLA, to protect the users' privacy by selecting the dummy locations to achieve k-anonymity according to personal visit probability for users' queries.

In the last article "FTS: A Feature-preserving Trajectory Synthesis Model", Li et al. present a novel feature-preserving model to generate new trajectories by extracting data features from the original dataset and validate the data by comparing the features of generated trajectories against the original dataset.

The special issue was preceded by the 18th Asia-Pacific Web International Conference (APWeb 2016), held at Suzhou. All the articles have undergone at least two rounds of rigorous peer-review according to the journal's high standards. We would like to thank all the reviewers involved for their invaluable input.

The guest editors believe the papers appearing in this issue form an accurate representation of current topics in the spatio-temporal data management and hope these articles will stimulate further development in this area. The editors express their appreciation to the authors and reviewers for contributing to this special issue.

We hope you enjoy this special issue and take some inspiration from it for your own future research. 